\title{
Using laser diffraction to measure agricultural sprays: Common sources of error when making measurements
}

\author{
W. Clint Hoffmann ${ }^{1 *}$, Bradley K. Fritz ${ }^{2}$, Yubin Lan ${ }^{3,4,5}$ \\ (1. Prology Consulting LLC, College Station, Texas, 77845, USA; \\ 2. USDA ARS Aerial Application Technology Research Unit, 3103 F\&B Road, College Station, Texas, 77845, USA; \\ 3. National Center for International Collaboration Research on Precision Agricultural Aviation Pesticides Spraying Technology/ \\ College of Engineering, South China Agricultural University, Guangzhou 510642, China; \\ 4. Department of Biological and Agricultural Engineering, Texas A\&M University, College Station, Texas, 77843, USA; \\ 5. Texas A\&M AgriLife Research and Extension Center, Beaumont, Texas, 77713, USA)
}

\begin{abstract}
In an agricultural setting, laser diffraction is a technique used to measure the size of particles, such as spray droplets or soil particles. Measurement of spray droplets allow users to create a desired droplet size through selection of spray nozzles, operating pressures, and adjuvants to maximize effectiveness of agrochemicals with minimum negative impact on the surrounding environment. The objective of this work is to provide practical guidance to new users of laser diffraction based on years of experience by the authors. The goal will be to highlight and discuss key issues to consider when making laser diffraction measurements, including proper setup and alignment of the laser, obscuration effects, background light scattering and other potential sources of error.
\end{abstract}

Keywords: Laser diffraction, droplet size, agricultural sprays

DOI: $10.33440 /$ j.ijpaa.20180101.0005

Citation: Hoffmann W C, Fritz B K, Lan Y B. Using laser diffraction to measure agricultural sprays: common sources of error when making measurements. Int J Precis Agric Aviat, 2018; 1(1): 15-18.

\section{Introduction}

Droplet size plays a very important role in the delivery and effectiveness of agrochemicals for plant protection. Ground and aerial applicators use a combination of nozzles, spray adjuvants, and operational settings, such as pressure, to create droplet sizes that will maximize efficacy for a particular spray application. Improper droplet size selection can lead to reduced performance of the agrochemical and spray drift. Laser diffraction is one of the most common tools to measure droplet size used by nozzle manufacturers, agrochemical and adjuvant producers, and researchers.

The basic principle behind laser diffraction is that light, i.e. a laser, is diffracted when it passes through a droplet and that the diffraction pattern is proportional to the diameter of the droplets. The two main optical theories that describe and predict this diffraction pattern are the Fraunhofer Diffraction ${ }^{[1]}$ and Mie scattering $^{[2,3]}$. Modern LD instruments use these theories to estimate the diameters of the spray droplets that pass through the laser beam. As there is extensive literature available detailing the mathematical theories and algorithms associated with laser diffraction $^{[4-6]}$, only a general overview will be presented. There are two main components to most laser diffraction instruments; the emitter, which emits the laser beam, and a receiver, which houses a series of 30 or more concentric photodetection cells, similar to rings on tree. When no spray droplets are present, the laser beam

Received date: 2018-05-10 Accepted date: 2018-09-18

Biographies: Bradley K. Fritz, PhD, Research Leader, research interests: agricultural aerial applications, Email: brad.fritz@ars.usda.gov; Yubin Lan, PhD, Professor, Director, research interests: precision agricultural aviation application, Email: ylan@scau.edu.cn.

*Corresponding author: W. Clint Hoffmann, $\mathrm{PhD}$, research interests: agricultural aerial applications. Prology Consulting LLC, College Station, TX 77845, USA. Tel: +1-979-777-0815, Email: clint.hoffmann@gmail.com. passes through the center of the ring and no measurement is made. But when a spray droplet passes through the laser beam, the beam is diffracted at an angle proportional to the radius of the droplet with "large" droplets having smaller angles of diffraction than "small" droplets. The diffracted beam triggers the photodetectors and a count is made on a particular cell that corresponds to droplets of a known diameter. After thousands to millions of droplets have been measured, a histogram is created of the droplet size distribution.

A major advantage of laser diffraction is the speed and repeatability of the measurements. For a typical agricultural spray testing setup, individual measurement replications can be completed in seconds with the results immediately available. There are several international standards ${ }^{[7-10]}$ detailing proper setup, operation, validation, and results interpretation that all users should reference and follow. The objective of this manuscript is to provide practical guidance to new users of LD based on years of experience by the authors and to highlight a number of areas to be aware of when making measurements and interpreting the results.

\section{Materials and methods}

Laser diffraction has proven a very robust and time effective tool for measuring agricultural sprays. Modern instruments have updated features such as self-alignment of the optics and more access to the data processing software code that further enhance usability and speed. However, like any scientific instrument improper setup and use can introduce errors. Also like many other instruments out there, LD measurements will generally always result in an answer, regardless of whether proper setup and operation procedures were followed. Therefore, the user must remain diligent and attentive during all phases of testing from setup to day-to-day-operations to data analyses.

\subsection{Testing setup}

Numerous standards provide guidance on proper setup and 
operation of LD instruments and should be followed. In a series of round-robin tests between three testing laboratories, Fritz et al. developed a set of guidelines that helped to greatly increased the repeatability of measurements made in both high- and low-speed wind tunnels for agricultural sprays. It was found that a sampling distance of 31 and $46 \mathrm{~cm}$ (12 and $18 \mathrm{in}$ ) for low and high airspeeds, respectively, minimized the sampling bias for the LD instrument used by the three labs. For low speed wind tunnels, it was recommended that the airspeed be set to $6.7 \mathrm{~m} / \mathrm{sec}(15 \mathrm{mph})$ to avoid temporal sampling bias that can occur with LD.

\subsection{Reference nozzles}

The reference nozzles specified by the standard ASABE S572 establish the boundaries between the different droplet size classifications and are defined based on the droplet size measured from a series of flat fan nozzles noted in the standard. The standard defines the flow rate each nozzle should achieve at the reference operating flowrate at a tolerance level of $+/-0.04 \mathrm{~L} / \mathrm{min}$. This high tolerance flowrate is intended to ensure that reference nozzle sets obtained by different parties, at different facilities and from different sources will atomize the spray the same way, in theory producing the same droplet size spectrums. In recent testing by the authors, a number of sets of high tolerance flowrated reference nozzles were obtained from the manufacturer as sets intended to be used as reference nozzles. Droplet sizing testing of these nozzles sets, following protocols set by Fritz et al. (2013), was conducted to determine droplet size variability between the sets. It was not surprising that there were significant differences (JMP, Student's LSD with Alpha=0.05) between volume diameters within nozzles types given that laser diffraction measurements are typically highly repeatable resulting in very low standard deviations to the mean (Table 1). Based on the results of this work, three sets were selected as "gold" standard reference nozzle sets and distributed amongst three cooperating laboratories. Unfortunately, there is no group or organization currently responsible for coordinating an effort to evaluate and supply droplet size tested reference nozzles. The authors suggest that those conducting nozzle droplet size testing studies obtain a number of each type of reference nozzle and conduct their own evaluations and identify sets of nozzles that are statistically similar and fall within the median range of the sizes measured.

\subsection{Obscuration}

Obscuration is the amount of light that is being diffraction and absorbed by the spray that is being measured. If no spray or other contaminants are presented, the obscuration rate will be $0 \%$. Most LD instruments are able to display the obscuration rate, as a percentage, while the spray is being measured. Our experience has shown that as obscuration rates start to increase above $25 \%$, the potential for erroneous measurements also increases. To help understand why this might occur, one can consider measuring the spray droplets from a flat fan nozzle. This nozzle generates a thin spray sheet that may only be $5 \%$ as thick as it is wide. If the laser has to pass through the horizontal chord of the spray, the increased numbers of droplets scattering the beam can significantly reduce the laser intensity reaching the detector which potentially biases the measurement results. The authors have learned to slightly twist a flat fan nozzle approximately $10^{\circ}$ from horizontal to reduce the obscuration rate. This slight rotation of the nozzle body still allows for complete sampling of the spray plume.

\subsection{Alignment}

Perhaps one of the greatest difficulties and sources of error with LD instruments can be proper alignment of the emitter and
Table 1 Volume diameters from five sets of precision flowrated ASABE S572 reference nozzle sets operated at the reference specified pressures ${ }^{*}$

\begin{tabular}{|c|c|c|c|c|c|c|c|}
\hline Nozzle & Number & DV0.1 & & DV0.5 & & DV0.9 & \\
\hline \multirow{5}{*}{11001} & 1 & $63.3 \pm 0.63$ & $\mathrm{~b}$ & $133.4 \pm 1.53$ & c & $225.3 \pm 2.46$ & C \\
\hline & 2 & $62.1 \pm 0.53$ & b & $129.9 \pm 0.89$ & b & $217.4 \pm 1.97$ & $\mathrm{~b}$ \\
\hline & 3 & $60.6 \pm 1.59$ & a & $127.1 \pm 3.27$ & $\mathrm{a}$ & $213.7 \pm 6.63$ & $\mathrm{a}$ \\
\hline & 4 & $63.4 \pm 0.19$ & b & $134.2 \pm 0.41$ & C & $227.4 \pm 0.78$ & C \\
\hline & 5 & $63.2 \pm 0.67$ & $\mathrm{~b}$ & $133.6 \pm 0.99$ & c & $226.2 \pm 1.75$ & C \\
\hline \multirow{5}{*}{11003} & 1 & $109.6 \pm 0.85$ & a & $241.2 \pm 2.00$ & $\mathrm{a}$ & $398.7 \pm 0.57$ & $\mathrm{a}$ \\
\hline & 2 & $110.5 \pm 0.49$ & b & $242.9 \pm 1.17$ & b & $400.1 \pm 1.46$ & $\mathrm{a}$ \\
\hline & 3 & $111.3 \pm 0.76$ & b & $245.3 \pm 0.88$ & c & $405.3 \pm 0.80$ & $\mathrm{~b}$ \\
\hline & 4 & $110.6 \pm 0.88$ & b & $244.2 \pm 2.34$ & c & $403.6 \pm 2.55$ & $\mathrm{~b}$ \\
\hline & 5 & $111.1 \pm 0.97$ & b & $243.8 \pm 0.60$ & c & $403.5 \pm 0.80$ & $\mathrm{~b}$ \\
\hline \multirow{5}{*}{11006} & 1 & $162.4 \pm 0.54$ & a & $352.0 \pm 1.20$ & $\mathrm{a}$ & $569.7 \pm 6.22$ & a \\
\hline & 2 & $170.1 \pm 0.93$ & c & $366.9 \pm 1.67$ & c & $587.0 \pm 0.95$ & C \\
\hline & 3 & $164.2 \pm 0.67$ & b & $355.8 \pm 0.73$ & b & $581.7 \pm 0.60$ & $\mathrm{~b}$ \\
\hline & 4 & $170.2 \pm 1.03$ & c & $369.8 \pm 1.24$ & c & $592.8 \pm 4.05$ & c \\
\hline & 5 & $164.6 \pm 0.69$ & $\mathrm{~b}$ & $356.4 \pm 0.70$ & b & $580.9 \pm 0.89$ & $\mathrm{~b}$ \\
\hline \multirow{5}{*}{8008} & 1 & $190.9 \pm 0.85$ & b & $424.9 \pm 1.57$ & b & $714.3 \pm 9.58$ & $\mathrm{~b}$ \\
\hline & 2 & $190.9 \pm 1.24$ & b & $427.0 \pm 1.14$ & b & $711.6 \pm 7.16$ & $\mathrm{a}$ \\
\hline & 3 & $191.2 \pm 1.10$ & b & $426.5 \pm 1.35$ & b & $724.5 \pm 4.90$ & b \\
\hline & 4 & $188.5 \pm 0.45$ & a & $423.0 \pm 1.60$ & a & $714.5 \pm 6.91$ & $\mathrm{~b}$ \\
\hline & 5 & $190.1 \pm 1.01$ & b & $425.5 \pm 1.73$ & $\mathrm{~b}$ & $718.4 \pm 2.45$ & $\mathrm{~b}$ \\
\hline \multirow{5}{*}{6510} & 1 & $223.8 \pm 0.96$ & a & $504.3 \pm 1.61$ & a & $856.1 \pm 9.39$ & $\mathrm{a}$ \\
\hline & 2 & $227.0 \pm 0.96$ & b & $507.6 \pm 1.79$ & b & $843.8 \pm 7.11$ & $\mathrm{a}$ \\
\hline & 3 & $228.7 \pm 1.22$ & c & $510.0 \pm 1.88$ & c & $853.2 \pm 7.22$ & $\mathrm{a}$ \\
\hline & 4 & $229.1 \pm 1.36$ & c & $512.5 \pm 2.06$ & c & $873.0 \pm 16.17$ & $\mathrm{~b}$ \\
\hline & 5 & $229.5 \pm 1.51$ & c & $512.1 \pm 3.08$ & c & $851.2 \pm 15.99$ & $\mathrm{a}$ \\
\hline \multirow{5}{*}{6515} & 1 & $315.1 \pm 1.52$ & c & $669.4 \pm 4.79$ & $\mathrm{a}$ & $1117.1 \pm 34.33$ & b \\
\hline & 2 & $317.4 \pm 1.60$ & c & $676.4 \pm 4.65$ & $\mathrm{~b}$ & $1145.3 \pm 28.28$ & c \\
\hline & 3 & $317.8 \pm 1.25$ & c & $680.1 \pm 3.54$ & $\mathrm{~b}$ & $1162.2 \pm 9.39$ & c \\
\hline & 4 & $312.4 \pm 2.00$ & b & $666.6 \pm 6.21$ & a & $1098.6 \pm 30.07$ & $\mathrm{a}$ \\
\hline & 5 & $306.1 \pm 3.53$ & $\mathrm{a}$ & $662.8 \pm 6.20$ & $\mathrm{a}$ & $1126.3 \pm 31.61$ & C \\
\hline
\end{tabular}

Note: ${ }^{*}$ DV0.1, DV0.5, and DV0.9 are the droplet diameters at which 10, 50 and $90 \%$, respectively, of the total spray volume is contained in droplets of lesser diameter.

receiver components. In a static laboratory setting, alignment can be mitigated by using optical rails and a dedicated setup. However, most agrochemical spray measurements are made in wind tunnels that may have multiple uses requiring the LD instrument to be moved in and out of the tunnel and potentially mounted onto separate stands requiring confirmation and adjustment of alignment each time the instrument is used. Most systems come with alignment tools that are used to adjust the system to ensure that the beam is passing through the center of the photodetector. Depending on a user's setup, this can be time-consuming process.

\subsection{Subtracting background noise}

When taking LD measurements, it is important to account for any background noise due to dust in the air or lens. A background measurement (also called reference or null measurement) taken before taking an actual measurement with the spray present can eliminate these errors. During this reference measurement, it is important for the user to watch the signal strength of the laser on the different measurement channels (Figure 1). There will often be some signal strength on the channels near the center of the photodetector (i.e. channels $0-2$ ); 
however, if the higher channels peaking, the user should check alignment and lens clarity.

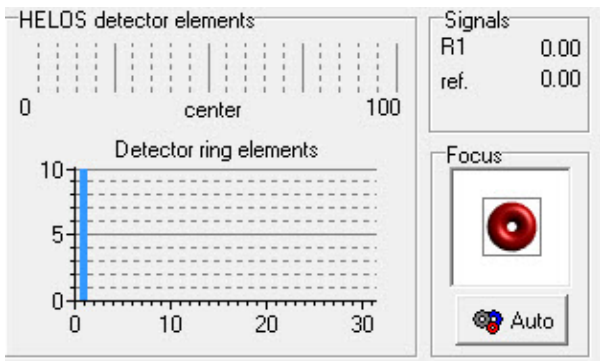

Figure 1 Reference measurement screen showing proper alignment of the laser diffraction instrument for a Sympatec Helos system

\section{Results and discussion}

Besides some of the physical setup issues discussed in the previous section, there are also issues related to user observations while the data is being collected and then post interpretation and analyses of the data. The number one thing that a user can do to prevent erroneous data is to simply pay attention during the measurement process. As many experiments may span several hours days or even weeks, it easy to become distracted and "just let the equipment run itself.” Users should constantly be watching the LD data collection screen and looking for some of the following items and their likely cause:

- Obscuration levels that are above 25\%: Too much spray is passing through the laser at one time;

- Reference measurements are increasing in signal strength: Lens contamination;

- All channels are indicating spray: The lens detection limits are too small for spray being measured so switch lens;

- High signal strength on the last channel: Vibrations are being detected or lens are contaminated (Figure 2).

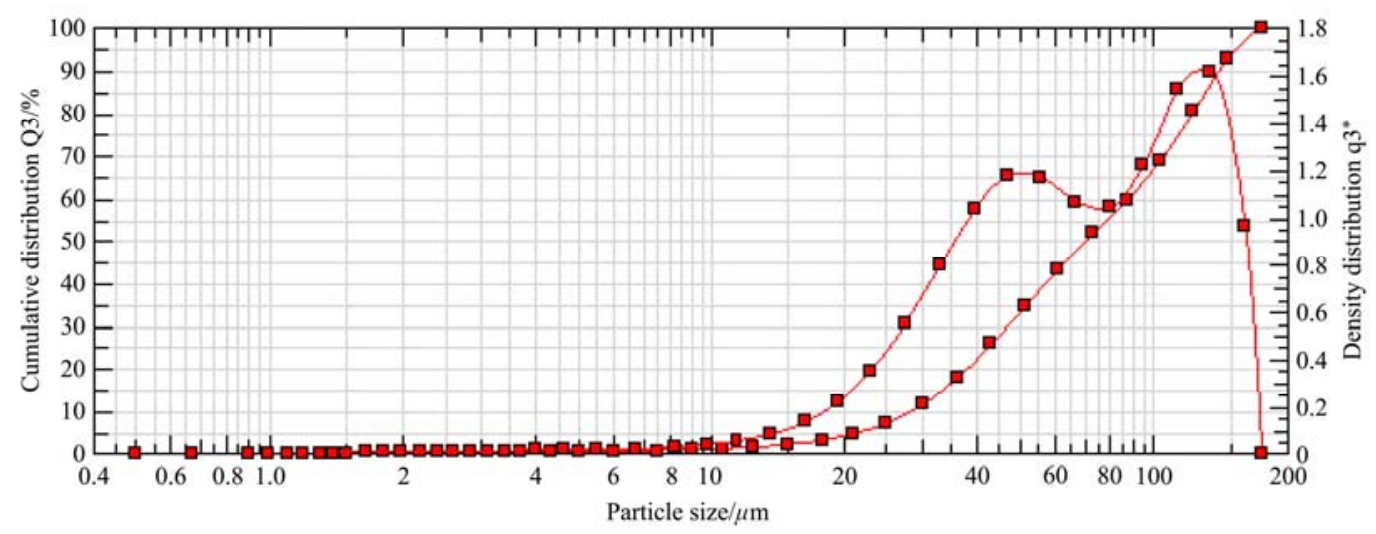

Figure 2 High signal strength on the highest channel (200 $\mu \mathrm{m})$ indicating vibration or lens contamination

After a replication, the user should check the results looking for consistency from replication to replication and troubleshooting if differences were seen where none were expected. The resulting spray distribution should be a single-peaked normal distribution and not a bimodal. Non-normal distributions can result from any of the items mentioned in the above list, as well as leaking nozzles, air in the spray line, and lens contamination during the spray tests. In some cases, these distributions can be caused by spray ligaments in the spray resulting from incomplete spray atomization. For some spray solution and nozzle combinations, complete spray atomization may not occur until distances greater than one meter from the nozzle, which would require increasing the distance between the nozzle and the measurement zone. This situation is common in high airspeed tests, such as those designed to simulate aerial application conditions. It should be noted that authors have experienced a few nozzles that do produce a bimodal distribution under very low or high spray pressures.

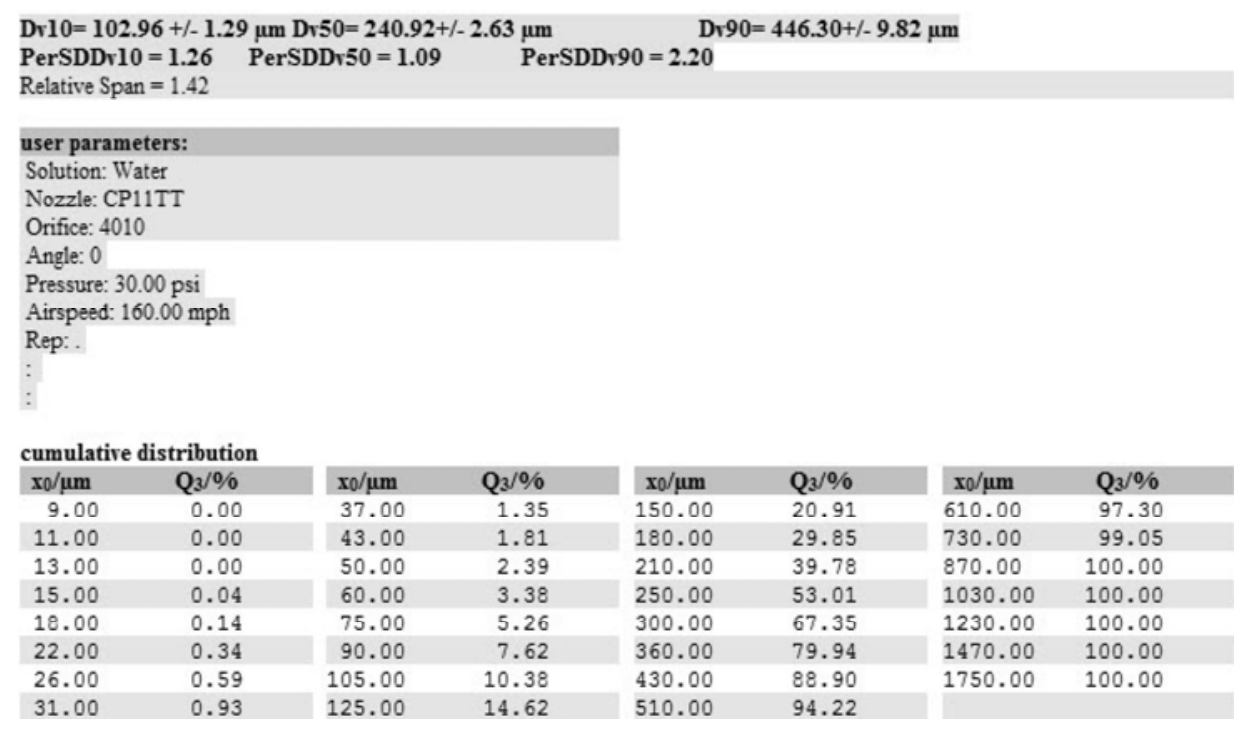

Figure 3 Results screens from a laser diffraction measurement showing means, standard deviation values, and spray volume within each measurement bin. Standard deviations below 5\% indicate consistent measurements between replications 
After a set of replications, the user should perform a quick analyses of the data to look at the standard deviation between replications. As a best management practice, the authors suggest that additional replications be performed if the standard deviation is greater than $5 \%$ for any of the droplet size statistics (such as volume median diameter) that the user is measuring and reporting. An example of the output screen showing the standard deviations for $\mathrm{Dv}_{0.1}, \mathrm{Dv}_{0.5}$, and $\mathrm{Dv}_{0.9}$ is shown in Figure 3 .

A final issue to consider is the precision of LD results which generally leads to very low standard deviations in the resulting data which further result in statistical differences being observed between treatments, even if numerical differences are minimal. While this is often the goal of the overall experiment, one should also consider the biological or real-world impact, or lack thereof, of these differences. It is common for measurements with just 2$4 \mu \mathrm{m}$ difference being statistically different. Users are therefore cautioned in the conclusions drawn from results of this type.

\section{Conclusions}

With the steady increase in laser diffraction systems being used in laboratories all over the world for research and evaluation of agricultural spray technologies, it is critical that those making the measurements stay vigilant and maintain good practices. There are several sources of error that can be manifested when making laser diffraction measurements, such as misalignment of the laser, vibrations, contaminated lens, and obscuration of the laser. If the user of a laser diffraction instrument does not watch for these items during the measurement, erroneous data will be collected and improper conclusions will be drawn from the results.

\section{Acknowledgments}

This work is supported by the 111 Project (D18019).

\section{[References]}

[1] Born M, Wolf E. Principles of Optics, 7th Edition. Cambridge University Press. 1999. ISBN 978-0-521-64222-4. 871 pp.

[2] van de Hulst H C). Light scattering by small particles. New York: John Wiley and Sons. 1957. ISBN 9780486139753. 470 pp.

[3] Hahn D W. "Light Scattering Theory" (PDF). 1999. University of Florida. Retrieved 2017-09-22.

[4] Dodge L G. Comparison of Performance of Droplet-Sizing Instruments. Appl. Optics, 1987; 26(7): 1328-1341.

[5] Teske M E, Thistle H W, Hewitt A J, Kirk I W. Conversion of Droplet Size Distributions from PMS Optical Array Probe to Malvern Laser Diffraction. Atomization Sprays, 2014; 24(9): 8.

[6] Tishkoff J M. Spray Characterization: Practices and Requirements. Opt. Eng., 1984; 23(5): 557-560.

[7] ASTM E1620-97(2016), Standard Terminology Relating to Liquid Particles and Atomization, ASTM International, West Conshohocken, PA, 2016, www.astm.org

[8] ASTM E799-03(2015), Standard Practice for Determining Data Criteria and Processing for Liquid Drop Size Analysis, ASTM International, West Conshohocken, PA, 2015, www.astm.org

[9] ISO 13320:2009, Particle Size Analysis - Laser Diffraction Methods.

[10] ASABE S572.1 Mar2009. Spray Nozzle Classification by Droplet Spectra. American Society of Agricultural and Biological Engineers. St. Joseph, MI

[11] Fritz B K, Hoffmann W C, Kruger G R, Henry R S, Hewitt A J, Czaczyk Z. Comparison of Drop Size Data from Ground and Aerial Application Nozzles at Three Testing Laboratories. Atomiz. Sprays 2014; 24(2): 181-192. 